\title{
Activation of neurotrophin signalling with light-inducible receptor tyrosine kinases
}

\author{
WEI ZHANG ${ }^{1 *}$, SHU ZHAO $^{2 *}$, LINJIE LU $^{3}$, ZHIMIN FAN ${ }^{1}$ and SHIXIN YE ${ }^{4,5}$ \\ ${ }^{1}$ Anesthesiology Department, Nanjing Hospital of Chinese Medicine Affiliated to Nanjing University of \\ Chinese Medicine, Nanjing, Jiangsu 210000; ${ }^{2}$ School of Life Science, Nantong University, Nantong, Jiangsu 226019 , \\ P.R. China; ${ }^{3}$ Institute of Genetics, Molecular and Cellular Biology, University of Strasbourg, Illkirch 67400; \\ ${ }^{4}$ Institut National de la Sante et de la Recherche Medicale (INSERM) U1195, Bicetre Hospital, \\ Paris-Saclay University, Le Kremlin-Bicêtre 94276; ${ }^{5}$ Laboratory of Computational and Quantitative \\ Biology (LCQB), Institute of Biology, Paris-Seine, Sorbonne University, Paris 75006, France
}

Received May 6, 2020; Accepted December 17, 2020

DOI: $10.3892 / \mathrm{mmr} .2022 .12586$

\begin{abstract}
Optogenetics combined with protein engineering based on natural light-sensitive dimerizing proteins has evolved as a powerful strategy to study cellular functions. The present study focused on tropomyosin kinase receptors (Trks) that have been engineered to be light-sensitive. Trk belongs to the superfamily of receptor tyrosine kinases (RTKs), which are single-pass transmembrane receptors that are activated by natural ligands and serve crucial roles in cellular growth, differentiation, metabolism and motility. However, functional variations exist among receptors fused with light-sensitive proteins. The present study proposed a signal transduction model for light-induced receptor activation. This model is based on analysis of previous light-induced Trk receptors reported to date and comparisons to the activation mechanism of natural receptors. In this model, quantitative differences on the dimerization induced from either top-to-bottom or bottom-to-up may lead to the varying amplitude of intracellular signals. We hypothesize that the top-to-bottom propagation is more favourable for activation and yields better results compared with the bottom-to-top direction. The careful delineation of the dimerization mechanisms
\end{abstract}

Correspondence to: Dr Zhimin Fan, Anesthesiology Department, Nanjing Hospital of Chinese Medicine Affiliated to Nanjing University of Chinese Medicine, 157 Daming Road, Qinhuai, Nanjing, Jiangsu 210000, P.R. China

E-mail: fanzm711@163.com

Dr Shixin Ye, Institut National de la Sante et de la Recherche Medicale (INSERM) U1195, Bicetre Hospital, Paris-Saclay University, 80 Rue du Gal Leclerc, Le Kremlin-Bicêtre 94276, France

E-mail: shixin.ye-lehmann@inserm.fr

*Contributed equally

Key words: neurotrophin signalling, tropomyosin kinase receptors, receptor tyrosine kinases neuronal growth factors, optogenetics fine-tuning activation will guide future design for an optimum cellular output with the precision of light.

\section{Introduction}

Cells respond to the environmental stimuli through a class of molecules on the cell surface called receptors. Receptor tyrosine kinases (RTKs) are a superfamily of receptors that were among the first receptors discovered and systematically studied; more importantly, the signalling pathway was one of the first pathways to unite various fields in developmental biology (1). Ligands of RTKs are protein molecules classified as growth factors. This family of ligands is active during embryonic development and in homeostasis in the adult body, activating a series of cell signalling pathways, serving pivotal roles in cell growth and differentiation. Research on RTK-related diseases, such as cancer, revealed that a number of acquired mutations in RTK receptors lead to the malfunctioning of the receptors (2).

There are 90 unique tyrosine kinase genes that have been identified in the human genome, of which 58 encode RTK proteins and the remaining 32 encode non-receptor types (1-5). The RTK family can be divided into 20 different types of receptors based on their ligands. Some of the well-known RTKs include tropomyosin receptor kinase (Trk) receptor, epidermal growth factor receptor (EGFR), platelet-derived growth factor receptor, fibroblast growth factor receptor, vascular endothelium growth factor receptor and insulin receptor kinase (IRK) Although RTK ligands are diverse, their activation mechanisms are highly conservative. After the extracellular portion of the receptor binds to the ligand, it activates the intracellular part of the receptor, which uses ATP as a phosphate donor to catalyse the transfer of phosphoryl groups onto specific tyrosine residues in the receptors.

It is now known that most RTK receptors are composed of a polypeptide chain and can be divided into three regions: i) A region to which the ligand binds from outside of the cell membrane; ii) a transmembrane region that crosses the cell membrane once; and iii) and the signal transduction region is the cytosolic side that contains the tyrosine kinase domain. In the absence of ligand, RTK exists in a monomeric state 
(Fig. 1). After binding to the ligand, RTK undergoes an oligomerization reaction (usually dimerization), which juxtaposes the tyrosine kinase domain in the cell membrane (4) to render the receptor active. For most RTKs, the generally accepted view is that this juxtaposition helps transactivation of tyrosine residues in the kinase activation loop or near the membrane region and induces conformational changes to stabilize the active state of the kinase (5). The resultant negatively charged phosphotyrosine residues subsequently recruit signalling proteins, such as through the Src homology 2 or phosphotyrosine binding domains of the signalling proteins, to activate the downstream signalling cascade $(6,7)$.

Following recent technological developments in proteomics and functional genomics, research on RTK signalling pathways has shifted from a study on isolated components in the ligand-receptor interaction to a broader understanding of the signalling pathway as an integrated system, including signalling proteins and the membrane environment. Such a system-wide analysis provided an unprecedented 'panoramic' view of tyrosine phosphorylation events (8).

The molecular weight range of the Trk receptor family is $140-145 \mathrm{kDa}$, and they all have glycosyl modifications. Neurotrophins are the most common ligands for Trk receptors, and they serve crucial roles in nervous system function (9). TrkA was the first member of the Trk receptor family to be discovered (5). During the discovery process, a fusion of tropomyosin to the kinase domain of TrkA was also identified, which was shown to be oncogenic and, therefore, TrkA was named tropomyosin kinase receptor $\mathrm{A}(8,9)$. A subsequent study isolated and identified two additional members of the family, TrkB and TrkC (10). Notably, all of these Trk genes are expressed in the nervous system $(9,10)$. In addition, their ligands are neurotrophins, and each receptor has a preference for a specific neurotrophin; for example, nerve growth factor (NGF) activates TrkA (10), brain-derived growth factor (BDNF) and neurotrophin (NT)-4/5 activates TrkB (11), and NT-3 activates TrkC. In a specific cellular environment, NT-3 can also activate both TrkA and TrkB (12).

However, alteration of ligand specificity of these receptors has been noted. For example, differential splicing of the Trk receptor genes results in the synthesis of proteins that have functionally essential differences in their extracellular domains (13). There is a fundamental difference in the function of receptors owing to splicing variants of the receptor genes. These splicing events are mainly divided into two categories: i) Splicing at the extracellular domain (13), such as the different isoforms of TrkA mentioned above; and ii) splicing at the kinase domain, such as the differential splicing found in TrkC that produces an isoform with a modified kinase domain with altered substrate specificity. Other examples are the isoforms of TrkB and TrkC that lack the kinase domain [as reviewed in (1)].

The most well-studied of Trk receptor is TrkA, owing to its association with NGF, which was the first discovered ligand of RTK receptor family. Before TrkA was identified (14,15), NGF had been extensively studied and was reported to exhibit a broad spectrum of neuronal functions, including neuronal survival, growth, differentiation, synapse formation and synaptic plasticity $(16,17)$. In the adult nervous system, NGF supports the maintenance and repair of nerves. Defects in NGF signalling are associated with a variety of neurodegenerative diseases. The currently accepted mechanism of TrkA activation is that the binding of NGF to the extracellular domain of the receptor results in phosphorylation of specific tyrosine residues in the phosphokinase region of the cytoplasmic domain (Fig. 1A) (1,5). These negatively charged phosphate groups then recruit signalling molecules and activate multiple signalling pathways, with Y490 and Y785 phosphorylation in the ICD involved in the activation of the Raf/MEK/ERK and PLC $\gamma$-PKC signalling pathways being the most well characterized (18) (Fig. 1E). After TrkA binds to NGF, the receptor complex together with their signalling molecule is internalized into cells either by the classical clathrin-mediated endocytosis $(19,20)$ or the pincher-mediated macrophage action (21). The fate of the receptor complex is less well understood, although previous studies have shown it may propagate the survival and differentiation signals through retrograde transport along the axon $(22,23)$. Therefore, comprehensive elucidation of the TrkA signalling should enable a better understanding of its function and life cycle.

Although there have been numerous studies on Trk signalling using TrkA as a model system, the lack of a method to accurately activate Trk receptor greatly limits our understanding of TrkA signalling and its dynamics, which further hinders the development of related treatment strategies. There is a lack of in-depth analysis in the following three aspects: First, although the identities of the downstream signalling effectors are known, it is extremely difficult to determine which signalling molecules are involved in transporting the TrkA signalling complex into the cell. Second, since the signalling of TrkA is dynamically regulated in time and space, how does the difference in cell membrane caused by subcellular environment affect signal specificity? Third, the intracellular kinase domain of TrkA consists of multiple phosphorylation sites, but how the phosphorylation of these sites regulates different signalling proteins and activates a specific pathway through coordination with each other is unclear. These problems all require the development of a proper tool to activate TrkA receptor with more precise spatial and temporal controls than their ligands relying on relatively slow diffusion and binding. The emergence of optogenetics, which is based on natural light-sensitive proteins, provides a new strategy for controlling protein function with high spatial and temporal resolution (24-26). To date, two types of light-sensitive proteins, the light-oxygen-voltage-sensing (LOV) domain and the cryptochrome 2 (CRY2), have been tested on TrkA receptors, providing new molecular tools to study intracellular signalling of this important family of receptors. The present study proposed a signal transduction model for light-induced receptor activation. This model is based on analysis of recently engineered light-induced Trk receptors reported to date and comparisons to the activation mechanism of natural receptors. The careful delineation of the dimerization mechanisms fine-tuning activation will guide future design for a specific cellular output using precise light.

\section{Materials and methods}

Analysis of structures. Crystal structures of TrkA and insulin receptor kinase (IRK) were chosen for analysis. Crystal structures were downloaded from the Research Collaboratory for Structural Bioinformatics Protein Data Bank (PDB; www.rcsb.org) for the inactive TrkA receptor kinase domain (PDB: 4GT5), the IRK in the inactive (PDB: 
A
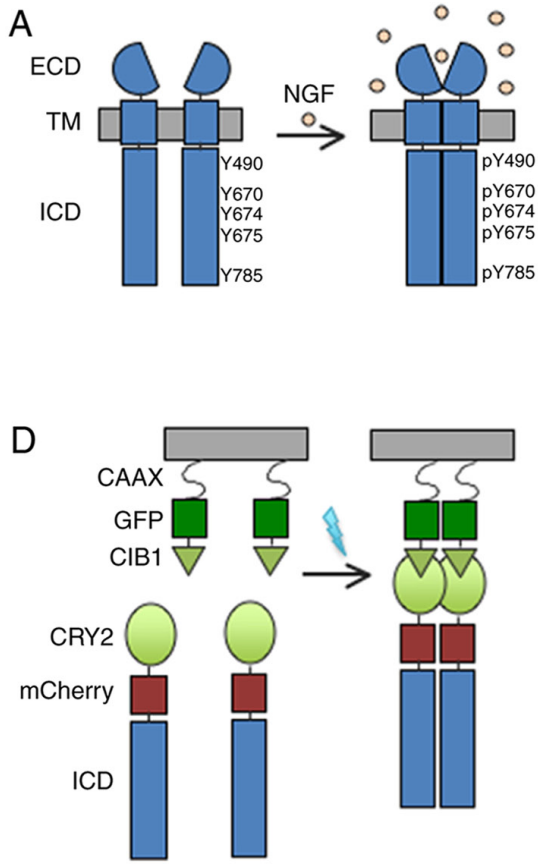

B

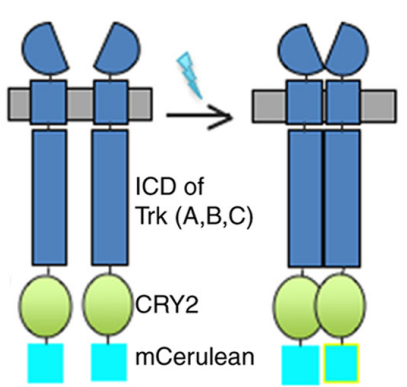

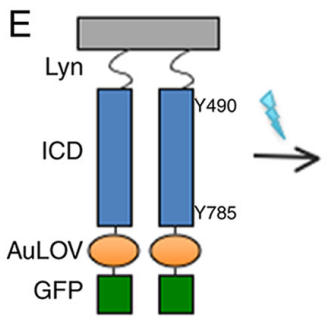

C

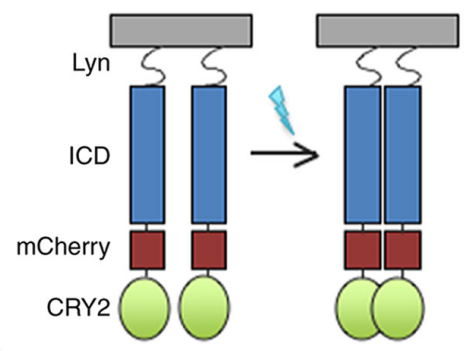

Figure 1. Schematic illustrations of wild-type TrkA and photoactivatable TrkA receptors. (A) TrkA has a single-helix TM domain that anchors the receptor at the plasma membrane. In the absence of ligand, TrkA exists in a monomeric state. NGF binds to the ECD to promote dimerization and phosphorylation of key tyrosines (Y490, Y670, Y674, Y675 and Y785) within the tyrosine kinase ICD and activates downstream pathways. (B) Full-length Trk (A, B or C) is linked to CRY2 and the monomeric cyan fluorescent protein mCerulean. This design appends on the photosensitive protein system, CRY2, which undergoes dimerization by blue light illumination. Similar to GFP, mCerulean was fused to the receptor to facilitate the characterization of receptor expression. (C) The ECD and TM were replaced by the lipidation Lyn moiety, but retained the normal plasma membrane activity. mCherry was fused to the receptor to facilitate the characterization of receptor expression. (D) The membrane targeting sequence Lyn is replaced with CIB1-GFP-CAAX. CIB1 is the interactive partner of CRY2. Light-sensitive TrkA is localized to the plasma membrane and dimerizes in a light-dependent manner. (E) Similar to the design of (C) in which the ECD and TM were replaced by the lipidation Lyn moiety to remove ligand binding activity, this design exploited the AuLOV domain for light induced dimerization. Blue light stimulation promotes intracellular dimerization of the receptor and activates downstream pathways. In this process, the phosphorylation of Y490 and Y785 in the ICD involved in the activation of the Raf/MEK/ERK and PLC $\gamma$-PKC signalling pathways, respectively. To characterize the receptor expression, GFP was also fused to the receptor. AuLOV, light-oxygen-voltage-sensing domain of aureochrome1 from Vaucheria frigida; CRY, cryptochrome 2; ECD, extracellular domain; GFP, green fluorescent protein; ICD, intracellular domain; NGF, nerve growth factor; p, phosphorylation; PLC, phosphoinositide phospholipase C- $\gamma$; PKC, protein kinase C; TM, transmembrane; Trk, tropomyosin receptor kinase; CIB1, calcium and integrin-binding 1.

1IRK) and phosphorylated/activated (PDB: 1IR3) state. PyMOL software (version 1.8) was used to display structures.

Analysis of experimental data. Functional assay readouts were extracted from previously published data (24-26), including ERK translation [also termed as ERK expression (26)], neurite growth and neuronal cell survival rate. Neurite growth assay (often referred as neurite outgrowth) quantifies the process wherein developing neurons produce new projections as they grow in response to guidance cues. The readouts were measured before and after light treatment conditions. The neurite growth and cell survival rate data were normalized using ratio of the number of surviving cells/total number of cells. Functional outputs are represented by the activities ratio, (readout obtained + light)/(readout obtained-light), measured in three types of assays: ERK translocation, neurite growth and neuron survival rate (24-26). Data are presented as the mean $\pm \mathrm{SD}$, $\mathrm{n} \geq 3$.

Statistical analysis. Statistical analyses were performed using StatPlus:mac software version 7 (AnalystSoft, Inc.). ANOVA followed by Tukey post-hoc test for pairwise multiple comparison procedure was performed. $\mathrm{P}<0.05$ was considered to indicate a statistically significant difference.

\section{Results and Discussion}

TrkA activation by light with spatial and temporal precision. Over the past five years, several Trk proteins have been engineered to respond to light (summarized in Fig. 1B-E and Table I). The first design was demonstrated in 2014 by Chang et al (24), who inserted the light-sensitive protein photolyase homology region (PHR) of CRY2 into the full-length Trk receptor sequence and also combined mCerulean (a cyan fluorescent protein) at the end of the PHR as the fluorophore (Fig. 1B). CRY2, together with CRY1, belongs to a class of flavoproteins found in plants and animals that respond to blue light. They regulate circadian rhythms and may also function in the sensing of magnetic fields in several species. To validate the functional expression of the light-sensitive Trks, intracellular ERK, $\mathrm{Ca}^{2+}$ indicator R-GECO1, AKT and EGFR inhibitor in signal activity were compared before and after light stimulations. Translocation ERK expression from the cytosol to nucleus was also measured by using fluoresce intensity and analysed by the nucleus/cytosol intensity ratio before and after light treatment. Dynamic axon movement was recorded by live video dark $400 \mathrm{sec}$ and light $370 \mathrm{sec}$. These functional outputs were summarized in Table II. Although this system was successful in controlling ERK activation and dynamic movement of the receptors along neurites using light, 


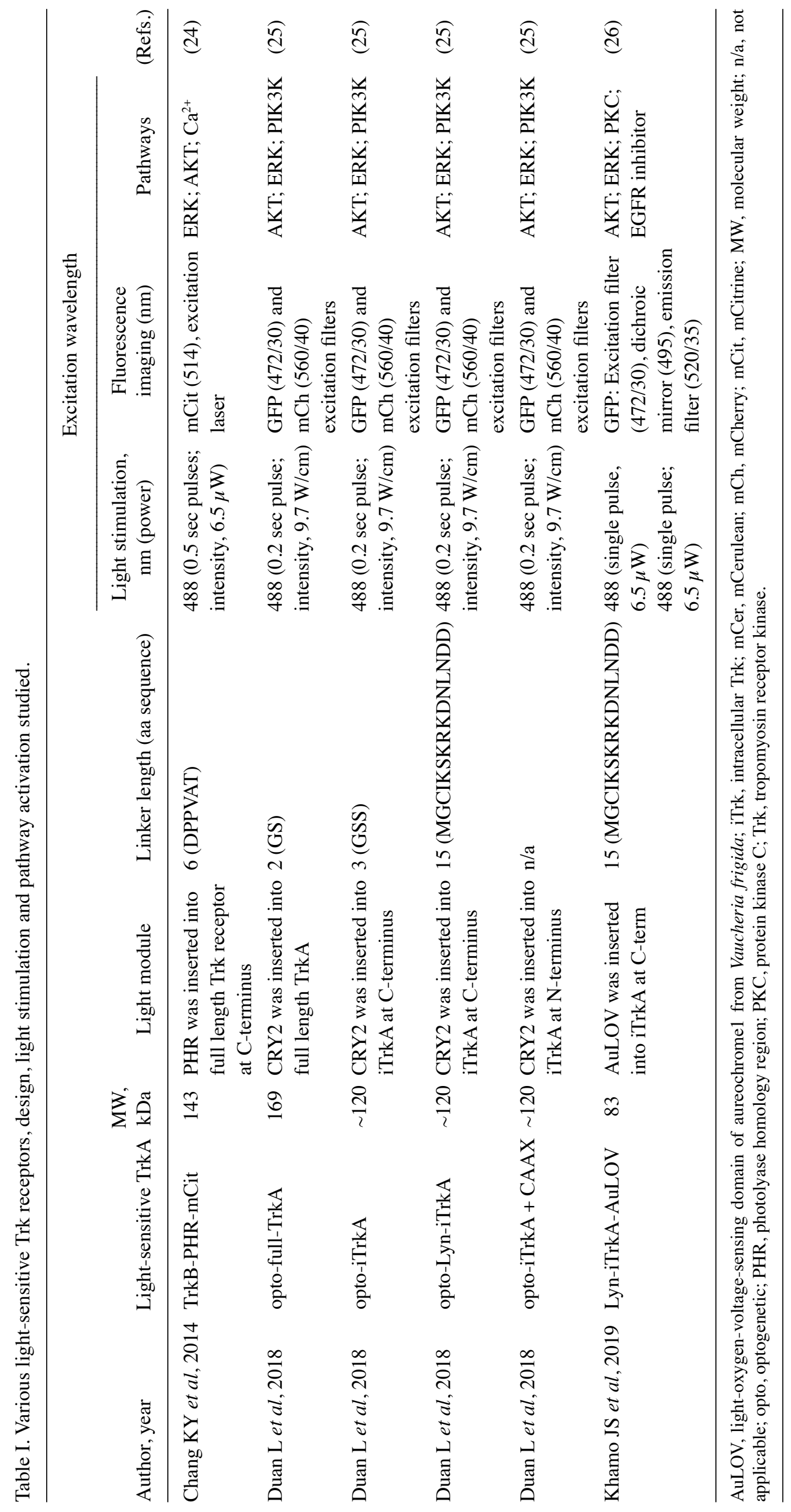


Table II. Functional output of light-sensitive Trk receptors.

Functional output $^{\mathrm{a}}$

\begin{tabular}{lcccc} 
Light-sensitive Trk & Propagation direction & ERK translocation & Neurite growth & Neuron survival rate $^{\mathrm{b}}$ \\
\hline TrkB-PHR-mCit & Bottom-to-top & 1.5 & 7 & $\mathrm{n} / \mathrm{a}$ \\
opto-full-TrkA & Bottom-to-top & 1.1 & 1 & $4.11 \pm 2.3$ \\
opto-iTrkA & Bottom-to-top & 1.25 & 5.48 & $12.72 \pm 3.4$ \\
opto-Lyn-iTrkA & Bottom-to-top & 1.5 & 1.69 & $27.14 \pm 14$ \\
Lyn-iTrkA-AuLOV & Bottom-to-top & 3 & 2 & $\mathrm{n} / \mathrm{a}$ \\
opto-iTrkA-CAAX & Top-to-bottom & 1.5 & 35.88 & $73 \pm 30$
\end{tabular}

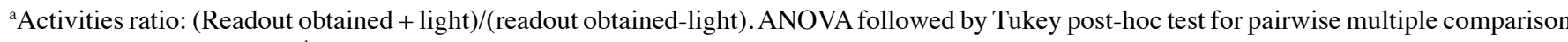
procedure was performed. ${ }^{\mathrm{b}} \mathrm{P}=0.0001$ between groups.

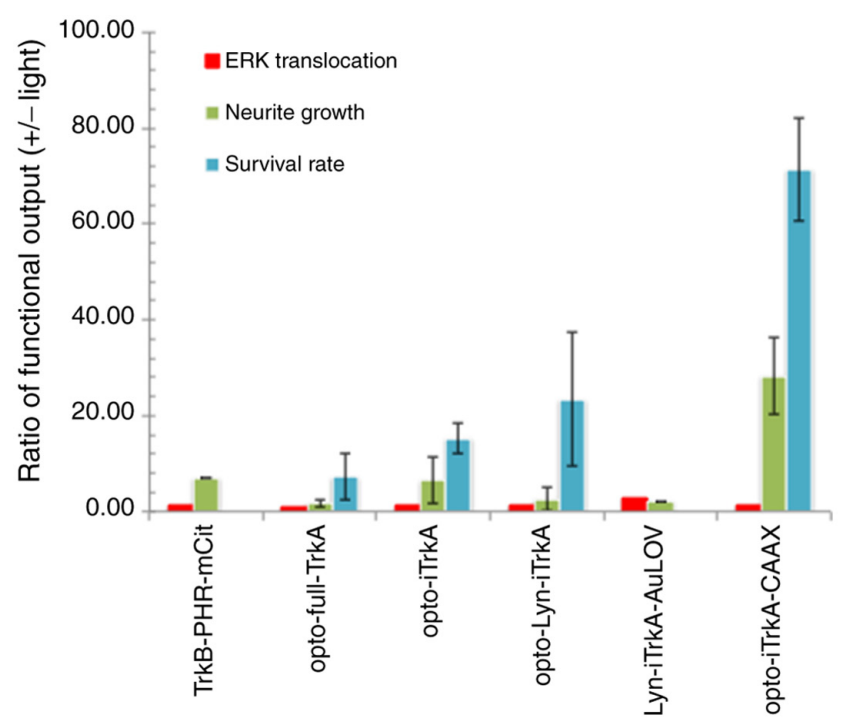

Figure 2. Summary of light-induced activities of various Trk constructs. Functional outputs are represented by the activities ratios (+/- light) measured in three types of assays: ERK translocation, neurite growth and neuron survival rate. See Duan et al (2018) (25) for details about experimental procedures of neuron survival rate, Khamo et al (2019) (26) and Chang et al (2014) (24) for details about ERK translocation and neurite growth. Data are presented in Table II. The top-to-bottom design (optoiTrkA+CAAX) has the best light-sensitivity among all constructs. For each condition, $n \geq 3$. ANOVA results for the survival rate: $n=3, F=34.83$; $\mathrm{P}=0.00006$.

there was high basal activity presumably due to the presence of ligand-banding domain in the Trk receptors.

In 2018, Duan et al introduced Arabidopsis thaliana CRY2 into intracellular TrkA (iTrkA) (25), a truncated receptor without the ligand-binding domain (Fig. 1C and D), which significantly reduced receptors' basal activity without light stimulation. Altogether, Duan et al made four different designs (Table I), i) opto-full-TrkA, ii) opto-iTrkA, iii) opto-Lyn-iTrkA, and iv) opto-iTrkA+CAAX. The first construct has a similar design as Chang et al (24), which has a fusion of CRY motif into the full-length receptor. The second construct has the ligand-binding domain removed, whereas the third and fourth construct have an $\mathrm{N}$-terminal membrane anchor (Lyn motif or CAAX motif) to localize the receptors onto the plasma membrane. Among these
A
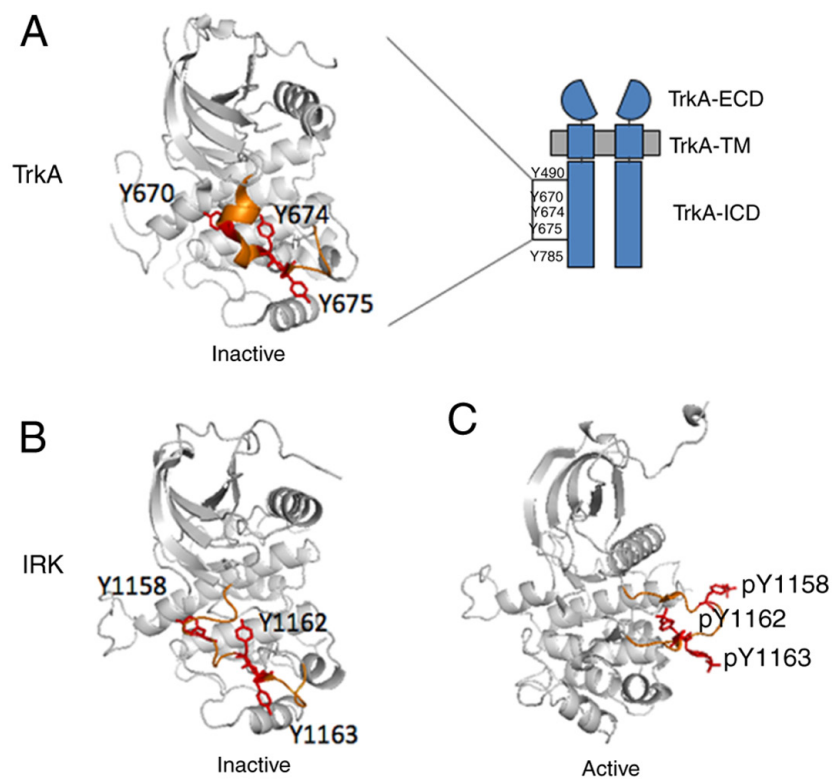

Figure 3. Crystal structures of TrkA and IRK tyrosine kinase domains. (A) Crystal structure of the TrkA tyrosine kinase domain (PDB: 4GT5) in the inactive state (37). The activation loop is coloured orange, with Y670, Y674 and Y675 residues coloured red. Crystal structures of the IRK in (B) the inactive (PDB: 1IRK) (28) and (C) in the activated (PDB: 1IR3) state (30). IRK crystal structures were adapted to depict graphically the TrkA tyrosine kinase domain in (A). The activation loop is coloured orange, with the homologous Y1158, Y1162, Y1163 residues coloured red. ECD, extracellular domain; ICD, intracellular domain; IRK, insulin receptor kinase; $p$, phosphorylation; TM, transmembrane; Trk, tropomyosin receptor kinase.

four different constructs, functional output before and after light activation was evaluated; these include ERK translocation, neurite growth and neuron survival rate. Even though all constructs demonstrated light-induced activities, the opto-iTrkA+CAAX design outperformed the other three constructs by demonstrating higher contrasts after light stimulation (Table II).

This approach was subsequently adapted to another light-sensitive protein containing the LOV domain (Fig. 1E; Tables I and II), which is a protein module present in many plants, microalgae, fungi and bacteria (25). This naturally occurring protein enables cells to sense environmental conditions and to control phototropism. Compared with CRY2 


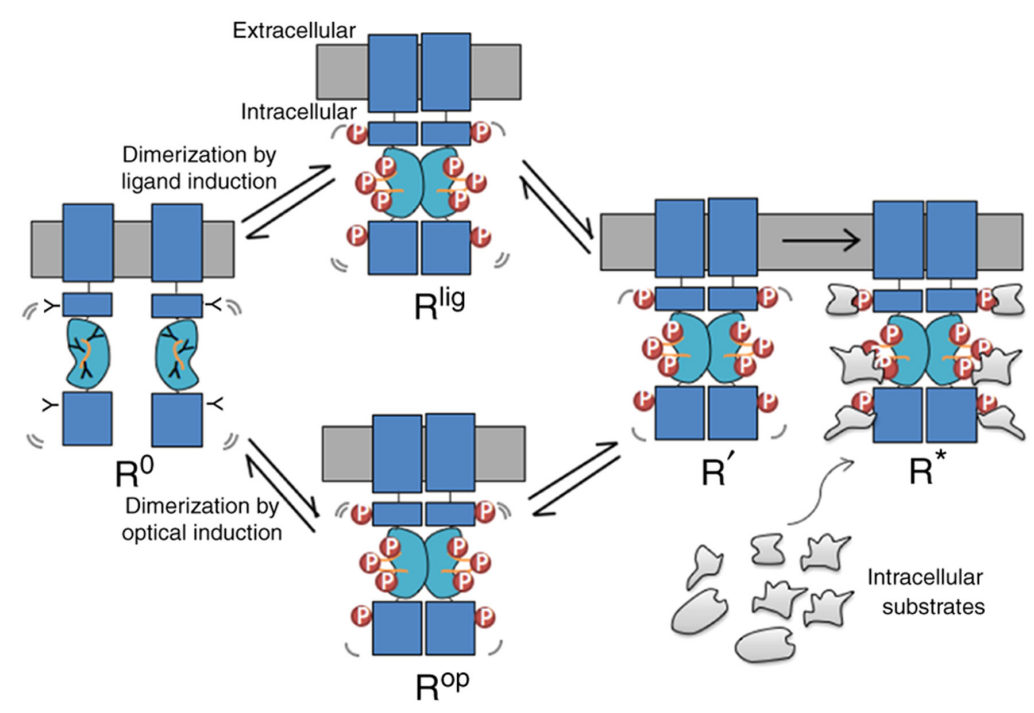

Figure 4. Light modules lend support to the dimerization model. RTKs exist in the monomeric ground state, $\mathrm{R}^{0}$. After binding to a ligand, or stimulated by the light, RTK dimerizes and reaches to the $\mathrm{R}^{\mathrm{lig}}$ or the $\mathrm{R}^{\mathrm{op}}$ state, respectively, with the intracellular kinase domains entering into a proper proximity such that tyrosines residues in the intracellular domain and activation loop (orange lines) are phosphorylated. Such kinase activation leads to the intermediate R' state, which subsequently creates docking sites for the recruitment of distinct intracellular in the activated $\mathrm{R}^{*}$ state. Lig, ligand; op, optogenetic; p, phosphorylation; RTK, receptor tyrosine kinase.

$(\sim 67 \mathrm{kDa})$, the LOV domain is a small, light-sensing module $(\sim 12-19 \mathrm{kDa})$. In this construct, Khamo et al (26) fused LOV domain of the aureochrome1 protein from the yellow-green alga Vaucheria frigida (AuLOV) with iTrkA. AuLOV responds to blue light and is excited at $488 \mathrm{~nm}$, the same wavelength as for CRY2. Following the design by Duan et al (25), Khamo et al (26) used the Lyn motif to position the receptor in the plasma membrane (Fig. 1E). The resultant light-sensitive Lyn-iTrkA-AuLOV was $83 \mathrm{kDa}$ the smallest in size among all designs. Khamo et al (26)demonstrated that activation of optogenetic TrkA significantly promoted cell differentiation. The present study summarized and analysed all the light-induced activities of the different constructs created and characterized from the three independent laboratories (Fig. 2; Table II). There is a general trend among the various functional assays performed that the neuron survival rate test has the highest sensitivity toward light stimulation. Furthermore, the construct (opto-iTrkA-CAAX) with a top-to-bottom design has the highest light sensitivity, manifested by the neuron survival rate assay (ANOVA; $\mathrm{F}=34.83 ; \mathrm{P}=0.00006$ ). Finally, various flexible linkers (Table I) inserted into the TrkA did not play significant roles in dictating the light-sensitivities of the receptors.

Light modules lend support to the dimerization model. The light-dependent alterations of the TrkA receptors analysed above, allow us to reach new conclusions regarding possible activation mechanisms in TrkA signalling when combined with structural information. In contrast with the extensive characterization of kinase domains of EGFR and insulin receptors (27-30), very little is known for the TrkA receptor. To date, there is only one isolated kinase domain of TrkA available (PDB: 4GT5), in which three of the tyrosines (Y670, Y674, Y675) could be located in the activation loop (highlighted in orange in Fig. 3A). This structure is considered to represent the inactive state because none of the tyrosine residues are phosphorylated. This makes it possible to analyse TrkA in the inactive state. In this structure, Y670 situates on a slightly more structured $\alpha$-helix compared with the Y674 and Y675 sites, which are both in the non-structured loop region. Inspired by the publication of Cunningham and Greene (27), which proposed TrkA kinase 3D structural models based on the insulin receptor IRK, the present study compared the available X-ray crystal structure of inactive TrkA kinase domain with the inactivated (PDB: 1IRK) (Fig. 3B) (28) and the activated (PDB: 1IR3) (Fig. 3C) (30) kinase domain of IRK. To the best of our knowledge, such TrkA structural comparison with IRK was not yet available. This analysis, for the first time, demonstrated that these domains both have the three activation loop phosphotyrosines (Y1158, Y1162, and Y1163) that are the direct homologous sequence of those in TrkA (Fig. 3). It was noticed that in IRK, the activation loop had undergone a significant conformational switch from inactivated to the activated state, exposing Y1158 (the equivalent of Y670 in TrkA) to the protein exterior in an entirely solvent-exposed environment, whereas the Y1162 (the equivalent of Y674 in TrkA) and Y1163 (the equivalent of Y675 in TrkA) remain relatively unchanged. Because the conservation of TrkA activation within the family of RTK, therefore, we hypothesized that the activation loop of TrkA may adopt conformational changes similar to what has been observed in IRK, which means that such change in the activation loop may promote dimerization of the kinase domain. A threshold model was proposed by Zinkle and Mohammadi (8) to explain RTK activation. In their model, they differentiate transit dimers and potent dimers. When RTK forms transit dimers, they are unable to phosphorylate kinetically disadvantaged sites, whereas RTK forms potent a dimer which enables specific downstream signals and influence the cellular response.

Based on above observations and comparisons, we propose a new light-induced activation model (Fig. 4) inspired by the photoreceptor rhodopsin, a G-protein-coupled receptor (31) and 
the ligand activated GPCR beta-adrenergic receptors (32). This model predicts that the unliganded receptor, which is defined as the $\mathrm{R}^{0}$ state that can undergo transitions through the stimulation of light, to the $\mathrm{R}^{*}$ state, which is defined as the active state. In this model, at least one intermediate state $\mathrm{R}^{\prime}$ is included, which is before the binding of the signalling molecules. $\mathrm{R}^{0}$ is stabilized by an unphosphorylated kinase domain, and intracellular substrates stabilize $\mathrm{R}^{*}$ through the interactions with the phosphorylated kinase domain. Mechanistically, this may be explained by considering that the $\mathrm{R}^{0}$ receptor (either un-liganded or un-induced by light) exists in a monomeric state, whereas $\mathrm{R}^{*}$ exists as oligomeric (dimer) state. Moreover, $\mathrm{R}^{0}$ may undergo spontaneous transitions to the $\mathrm{R}^{\prime}$ state, explaining the high basal activity observed for some TrkA constructs when the extracellular ligand-binding domain was removed. As shown in Fig. 3, binding of a ligand is similar to the light-induced activation that promotes kinase domain dimerization to occur sequentially, resulting in a series of conformational states that are intermediates ( $\mathrm{R}^{\text {lig }}$ and $\mathrm{R}^{\mathrm{op}}$. Subscript 'lig' refers to the ligand induced activation and 'op' refers to optogenetic activation.). Here, it is necessary to point out a factor that has been overlooked between the $\mathrm{R}^{\mathrm{lig}}$ and $\mathrm{R}^{\mathrm{op}}$ state, which is the direction of dimerization induction. For the ligand induction, the driving forces always come from the top of the receptors (induced from the extracellular side). However, for the light induction, the driving forces may come either from the top or bottom of the receptor, depending where the light-dependent module was fused into. From the experimental analyses, aforementioned, results from light-engineered TrkA receptors indicate that dimerization promoted from top-to-bottom direction are more efficient than bottom-to-top direction, as compared among four different TrkA CRY2 fusion constructs (especially between CAAX-CRY2-mCh-iTrkA vs. Lyn-iTrkA-CRY2; Fig. 2; Table II). Following the initial dimerization of the light-sensitive protein module (PHR, CRY2 or AuLOV), the kinase domain may undergo rearrangement and conformation changes (Fig. 3) that lead to autophosphorylation and dimerization. Each phosphorylation on the kinase domain stabilizes the dimer and depending on its conformation preferably recruits an intracellular substrate until the receptor has been stabilized by interactive proteins to reach the active $\mathrm{R}^{*}$ state. Any agents that can stabilize the $\mathrm{R}^{0}$ state can reverse the process and dephosphorylate the activate $\mathrm{R}^{\prime}$ state of the receptor. Conversely, some ligands may promote stronger dimers than other ligands, and some RTK mutants may stabilize unique conformational states having a lower affinity for the intracellular interactive proteins. Techniques that rely on the genetic code expansion (33), which allows the site-specific incorporation of unnatural amino acids into proteins, can potentially enable smaller modifications of the protein. Recently, we have successfully demonstrated the feasibility of controlling TrkA activity using genetic code expansion technique (34). Another important alternative strategy to reengineering kinase activity is through chemical genetics. Several recent reviews have summarized topics related to engineering at the gene, protein and ligand level (35-37), as well as the eukaryotic model organisms (38) in which the technique can be implemented. These strategies also have not yet been applied to the RTK receptors. The exploitation of these alternative approaches to engineer light-sensitive RTKs will certainly contribute to the future studies of RTK receptors and associated therapeutic strategies.
Finally, it was noted that the use of light-sensitive TrkA has thus been restricted to cells in tissue culture. However, optogenetics can also been applied in vivo. In the future, it may be possible to apply these the most robust light-sensitive TrkA in a living animal and to determine the nature of the tissue specific functions of TrkA in various cell types and organs. The major challenge associated with these experiments would be achieving effective photoactivation; for example, by using a transparent animal such as zebrafish or by surgically implanting a light emitter.

\section{Acknowledgements}

The authors thank Professor Michael Schumacher (INSERM U1195) for valuable suggestions.

\section{Funding}

Financial supports were made available by Sorbonne University start-up fund INRA S18LRPV022 and CNRS PEPS (grant: Exomod) to S.Y.

\section{Availability of data and materials}

The datasets used and/or analyzed during the current study are available from the corresponding author on reasonable request.

\section{Authors' contributions}

SY monitored the project progression, data analysis, interpretation, writing and editing the manuscript. WZ, LL, ZF and SZ contributed to data analysis. SY and LL prepared the initial draft of the manuscript; ZF and SY revised and finalized the manuscript. All authors reviewed, read and approved the final manuscript.

\section{Ethics approval and consent to participate}

Not applicable.

\section{Patient consent for publication}

Not applicable.

\section{Competing interests}

The authors declare that they have no competing interests.

\section{References}

1. Lemmon MA and Schlessinger J: Cell signalling by receptor tyrosine kinases. Cell 141: 1117-1134, 2010.

2. Sangwan V and Park M: Receptor tyrosine kinases: Role in cancer progression. Curr Oncol 13: 191-193, 2006.

3. Robinson DR, Wu YM and Lin SF: The protein tyrosine kinase family of the human genome. Oncogene 19: 5548-5557, 2000.

4. Fraser J, Cabodevilla AG, Simpson J and Gammoh N: Interplay of autophagy, receptor tyrosine kinase signalling and endocytic trafficking. Essays Biochem 61: 597-607, 2017.

5. Hubbard SR: Juxtamembrane autoinhibition in receptor tyrosine kinases. Nat Rev Mol Cell Biol 5: 464-471, 2004.

6. Pawson T, Gish GD and Nash P: SH2 domains, interaction modules and cellular wiring. Trends Cell Biol 11: 504-511, 2001. 
7. Ren S, Yang G, He Y, Wang Y, Li Y and Chen Z: The conservation pattern of short linear motifs is highly correlated with the function of interacting protein domains. BMC Genomics 9: 452, 2008.

8. Zinkle A and Mohammadi M: A threshold model for receptor tyrosine kinase signaling specificity and cell fate determination. F1000Res 7: 872, 2018.

9. Ip NY, Stitt TN, Tapley P, Klein R, Glass DJ, Fandl J, Greene LA Barbacid M and Yancopoulos GD: Similarities and differences in the way neurotrophins interact with the Trk receptors in neuronal and nonneuronal cells. Neuron 10: 137-149, 1993.

10. Brodeur GM, Nakagawara A, Yamashiro DJ, Ikegaki N, Liu XG Azar CG, Lee CP and Evans AE: Expression of TrkA, TrkB and TrkC in human neuroblastomas. J Neurooncol 31: 49-55, 1997.

11. Marshall J, Szmydynger-Chodobska J, Rioult-Pedotti MS, Lau K, Chin AT, Kotla SKR, Tiwari RK, Parang K, Threlkeld SW and Chodobski A: TrkB-enhancer facilitates functional recovery after traumatic brain injury. Sci Rep 7: 10995, 2017.

12. Zhou XF and Rush RA: Functional roles of neurotrophin 3 in the developing and mature sympathetic nervous system. Mol Neurobiol 13: 185-197, 1996.

13. Segal RA: Selectivity in neurotrophin signalling: Theme and variations. Annu Rev Neurosci 26: 299-330, 2003.

14. Kaplan DR, Martin-Zanca D and Parada LF: Tyrosine phosphorylation and tyrosine kinase activity of the trk proto-oncogene product induced by NGF. Nature 350: 158-160, 1991.

15. Klein R, Conway D, Parada LF and Barbacid M: The trkB tyrosine protein kinase gene codes for a second neurogenic receptor that lacks the catalytic kinase domain. Cell 61: 647-656, 1990.

16. Zhang Y, Moheban DB, Conway BR, Bhattacharyya A and Segal RA: Cell surface Trk receptors mediate NGF-induced survival while internalized receptors regulate NGF-induced differentiation. J Neurosci 20: 5671-5678, 2000.

17. Ninan I: Synaptic regulation of affective behaviors; role of BDNF. Neuropharmacology 76: 684-695, 2014.

18. Biarc J, Chalkley RJ, Burlingame AL and Bradshaw RA: Dissecting the roles of tyrosines 490 and 785 of TrkA protein in the induction of downstream protein phosphorylation using chimeric receptors. J Biol Chem 288: 16606-16618, 2013.

19. Beattie EC, Howe CL, Wilde A, Brodsky FM and Mobley WC: NGF signals through TrkA to increase clathrin at the plasma membrane and enhance clathrin-mediated membrane trafficking. J Neurosci 20: 7325-7333, 2000

20. Howe CL, Valletta JS, Rusnak AS and Mobley WC: NGF signaling from clathrin-coated vesicles: Evidence that signaling endosomes serve as a platform for the Ras-MAPK pathway. Neuron 32: 801-814, 2001.

21. Shao Y, Akmentin W, Toledo-Aral JJ, Rosenbaum J, Valdez G, Cabot JB, Hilbush BS and Halegoua S: Pincher, a pinocytic chaperone for nerve growth factor/TrkA signaling endosomes. J Cell Biol 157: 679-691, 2002.

22. Riccio A, Pierchala BA, Ciarallo CL and Ginty DD: An NGF-TrkA-mediated retrograde signal to transcription factor CREB in sympathetic neurons. Science 227: 1097-1100, 1997.
23. Bhattacharyya A, Watson FL, Bradlee TA, Pomeroy SL, Stiles CD and Segal RA: Trk receptors function as rapid retrograde signal carriers in the adult nervous system. J Neurosci 17: 7007-7016, 1997.

24. Chang KY, Woo D, Jung H, Lee S, Kim S, Won J, Kyung T, Park H, Kim N, Yang HW, et al: Light-inducible receptor tyrosine kinases that regulate neurotrophin signalling. Nat Commun 5: 4057, 2014.

25. Duan L, Hope JM, Guo S, Ong Q, François A, Kaplan L, Scherrer G and Cui B: Optical activation of TrkA signaling. ACS Synth Biol 7: 1685-1693, 2018.

26. Khamo JS, Krishnamurthy VV, Chen Q, Diao J and Zhang K: Optogenetic delineation of receptor tyrosine kinase subcircuits in PC12 cell differentiation. Cell Chem Biol 26: 400-410.e3, 2019.

27. Cunningham ME and Greene LA: A function-structure model for NGF-activated TRK. EMBO J 17: 7282-7293, 1998.

28. Hubbard SR, Wei L, Ellis L and Hendrickson WA: Crystal structure of the tyrosine kinase domain of the human insulin receptor. Nature 372: 746-754, 1994.

29. Artim SC, Mendrola JM and Lemmon MA: Assessing the range of kinase autoinhibition mechanisms in the insulin receptor family. Biochem J 448: 213-220, 2012.

30. Hubbard SR: Crystal structure of the activated insulin receptor tyrosine kinase in complex with peptide substrate and ATP analog. EMBO J 16: 5572-5581, 1997.

31. Fahmy K, Siebert F and Sakmar TP: Photoactivated state of rhodopsin and how it can form. Biophys Chem 56: 171-178, 1995.

32. Gether G and Kobilka BK: G protein-coupled receptors. II. Mechanism of agonist activation. J Biol Chem 273: 17979-17982, 1998.

33. Chen Y, Lu L and Ye S: Genetic code expansion and optoproteomics. Yale J Biol Med 90: 599-610, 2017.

34. Zhao S, Shi J, Yu G, Li D, Wang M, Yuan C, Zhou H, Parizadeh A, Li Z, Guan MX and Ye S: Photosensitive tyrosine analogues unravel site-dependent phosphorylation in TrkA initiated MAPK/ERK signaling. Commun Biol 3: 706, 2020.

35. Leopold AV, Chernov KG and Verkhusha VV: Optogenetically controlled protein kinases for regulation of cellular signaling. Chem Soc Rev 47: 2454-2484, 2018.

36. Islam K: The bump-and-hole tactic: Expanding the scope of chemical genetics. Cell Chem Biol 25: 1171-1184, 2018.

37. McCormick JW, Pincus D, Resnekov O and Reynolds KA: Strategies for engineering and rewiring kinase regulation. Trends Biochem Sci 45: 259-271, 2020.

38. Ryu J and Park SH: Simple synthetic protein scaffolds can create adjustable artificial MAPK circuits in yeast and mammalian cells. Sci Signal 383: ra66, 2015.

(i) $(-)$ This work is licensed under a Creative Commons Attribution-NonCommercial-NoDerivatives 4.0 International (CC BY-NC-ND 4.0) License. 